\title{
Towards quantized complex numbers: q-deformed Gaussian integers and the Picard group
}

\author{
Valentin Ovsienko ${ }^{1}$ \\ ${ }^{1}$ Centre National de la Recherche Scientifique, Laboratoire de Mathématiques de Reims, \\ UMR9008 CNRS, Université de Reims Champagne-Ardenne, U.F.R. Sciences Exactes et \\ Naturelles, Moulin de la Housse - BP 1039, 51687 Reims cedex 2, France, \\ valentin.ovsienko@univ-reims.fr
}

Received Date May 14, 2021; Accepted Date July 26, 2021

\begin{abstract}
This work is a first step towards a theory of " $q$-deformed complex numbers". Assuming the invariance of the $q$-deformation under the action of the modular group I prove the existence and uniqueness of the operator of translations by $i$ compatible with this action. Obtained in such a way $q$-deformed Gaussian integers have interesting properties and are related to the Chebyshev polynomials.
\end{abstract}

\section{Introduction}

The notion of $q$-deformed rational numbers was introduced in [11]. It was further extended to arbitrary real numbers in [12. Several properties of $q$-numbers were studied in [13, 8, 9$]$. The unimodality conjecture formulated in [11] was tackled in [10].

The goal of this paper is to extend the $q$-deformation to complex numbers. We show that this can be done in a unique way. Already for the simplest case of Gaussian integers, i.e., complex numbers with integer real and imaginary parts, the obtained $q$-deformation has quite nontrivial properties. In particular, we observe an unexpected relation with the Chebyshev polynomials.

We start the introduction by explaining in Sections 1.1 1.3 the approach that was used to define the notion of $q$-rationals. The role of the modular group $\operatorname{PSL}(2, \mathbb{Z})$ is crucial. Section 1.6 explains the main idea of this work.

\subsection{Euler's $q$-integers and the matrices $T_{q}$ and $S_{q}$}

The notion of $q$-deformed integers

$$
[n]_{q}:=\frac{1-q^{n}}{1-q}
$$

(C) The author(s). Distributed under a Creative Commons Attribution 4.0 International License 
where $n \in \mathbb{Z}$, goes back to Euler. The expression (11) reads for $n \geq 0$

$$
[n]_{q}=1+q+q^{2}+\cdots+q^{n-1}, \quad[-n]_{q}=-q^{-1}-q^{-2}-\cdots-q^{-n} .
$$

Definition (1) was used by Gauss to define $q$-binomial coefficients that now play an important role in combinatorics (see [18]), quantum algebra and quantum calculus (see [6]).

Euler's $q$-integers (1) can be characterized by the recurrence

$$
[n+1]_{q}=q[n]_{q}+1
$$

and the initial condition $[0]_{q}=0$.

Consider the standard action of the modular group $\operatorname{PSL}(2, \mathbb{Z})$ on the space of rational functions in $q$ defined by linear-fractional transformations. More precisely, elements of $\operatorname{PSL}(2, \mathbb{Z})$ are represented by $2 \times 2$ matrices acting on the rational function $X=X(q)$ via

$$
\left(\begin{array}{ll}
a & b \\
c & d
\end{array}\right) X=\frac{a X+b}{c X+d}
$$

Recurrence (2) describes an action of the subgroup of integer translations; this group is isomorphic to $\mathbb{Z}$ and generated by the matrix

$$
T_{q}=\left(\begin{array}{ll}
q & 1 \\
0 & 1
\end{array}\right) .
$$

The matrix $T_{q}$ acts on the function $X(q)$ via $T_{q} X=q X+1$, and recurrence (2) can be understood as the equivariance property $[T(n)]_{q}=T_{q}\left([n]_{q}\right)$, where $T$ is the translation operator $T(x)=x+1$, (see formula (6) below).

Furthermore,

$$
S_{q}=\left(\begin{array}{cc}
0 & -1 \\
q & 0
\end{array}\right)
$$

is the unique matrix that interchanges the "smallest" $q$-integers $[-1]_{q}$ and $[1]_{q}$. Therefore, the matrices $T_{q}$ and $S_{q}$ appear already at the level of $q$-integers. They play a crucial role in our approach.

Remark 1.1. Note that the matrix $S_{q}$ is widely known in the physics literature under the name of "spinor metric"; see, e.g., [20]. It can be used to characterize the usual quantum group $\mathrm{SL}_{q}(2)$ as the group of symmetry of this metric. However, I did not find in the literature a simultaneous use of the matrices $T_{q}$ and $S_{q}$ that generate a $\operatorname{PSL}(2, \mathbb{Z})$-action.

\subsection{The modular group action}

The modular group $\operatorname{PSL}(2, \mathbb{Z})$ has the standard generators

$$
T=\left(\begin{array}{ll}
1 & 1 \\
0 & 1
\end{array}\right), \quad S=\left(\begin{array}{cc}
0 & -1 \\
1 & 0
\end{array}\right)
$$


satisfying the relations $S^{2}=(T S)^{3}=\mathrm{Id}$. It transitively acts on $\mathbb{Q}$, via linear-fractional transformations.

The $q$-deformation (4)-(5) preserves the relations between the generators:

$$
S_{q}^{2}=\mathrm{Id}, \quad\left(T_{q} S_{q}\right)^{3}=\mathrm{Id} .
$$

as elements of $\operatorname{PSL}(2, \mathbb{Z}[q])$. Moreover, if $T_{q}$ is chosen to be as in (44), then the matrix $S_{q}$ can be characterized as the unique matrix satisfying (77). Therefore, $T_{q}$ and $S_{q}$ generate an action of the same, "undeformed", group $\operatorname{PSL}(2, \mathbb{Z})$ by linear-fractional transformations on the space $\mathbb{Z}(q)$ of rational functions $X=X(q)$ :

$$
T_{q} X=q X+1, \quad S_{q} X=-\frac{1}{q X} .
$$

It follows that, replacing $T$ by $T_{q}$ and $S$ by $S_{q}$, then every $A \in \operatorname{PSL}(2, \mathbb{Z})$ correctly defines a unique matrix $A_{q}$ with coefficients polynomially depending on $q$.

\subsection{Modular invariance characterizes $q$-rationals}

The notion of q-rationals was defined in [11] in a combinatorial way. It became clear later (see [8, 13, 9]) that the simplest, and perhaps most conceptual, way to define $q$-deformed rationals is to assume the modular invariance.

There exists a unique map

$$
\mathbb{Q} \longrightarrow \mathbb{Z}(q), \quad x \mapsto[x]_{q},
$$

that commutes with the $\operatorname{PSL}(2, \mathbb{Z})$-action and sends 0 to 0 .

Equivalently, the $q$-deformation $[x]_{q}$ of every $x \in \mathbb{Q}$ satisfies the following two linear recurrences

$$
[x+1]_{q}=q[x]_{q}+1, \quad\left[-\frac{1}{x}\right]_{q}=-\frac{1}{q[x]_{q}} .
$$

They express the invariance of the $q$-deformation under the action of the generators of $\operatorname{PSL}(2, \mathbb{Z})$.

Since $\operatorname{PSL}(2, \mathbb{Z})$ acts transitively on $\mathbb{Q}$, the requirement of $\operatorname{PSL}(2, \mathbb{Z})$-invariance implies the uniqueness of the rational function $[x]_{q}$. However, the existence of such a $q$-deformation (and its extension to $\mathbb{R}$; see [12]) is a non-trivial and quite remarkable fact.

\subsection{The starting point: elliptic points of $\operatorname{PSL}(2, \mathbb{Z})$}

Despite the fact that $\operatorname{PSL}(2, \mathbb{Z})$ consists of real matrices, the assumption of $\operatorname{PSL}(2, \mathbb{Z})$ invariance provides us with an infinite set of $q$-deformed complex numbers.

Let $x \in \mathbb{C}$ be a fixed point of some element $A \in \operatorname{PSL}(2, \mathbb{Z})$. Such points are called elliptic and their classification is well-known; see, e.g., [17]. The invariance condition then reads

$$
[A(x)]_{q}=A_{q}\left([x]_{q}\right)=[x]_{q},
$$

so that $[x]_{q}$ is a fixed point of $A_{q}$. For instance, $\pm i$ are fixed points of $S$, therefore $[ \pm i]_{q}$ are fixed points of $S_{q}$. One then obtains

$$
[i]_{q}:=\frac{i}{q^{\frac{1}{2}}}, \quad[-i]_{q}:=-\frac{i}{q^{\frac{1}{2}}} .
$$




\subsection{The space of rational functions in $q^{\frac{1}{2}}$}

It follows from (9) that, in order to consider $q$-deformed complex numbers, one needs to extend the space of rational functions in $q$ to the space $\mathbb{C}\left(q^{\frac{1}{2}}\right)$ of rational functions in $q^{\frac{1}{2}}$. This is the space we will work with, the action of all of the operators and groups that we consider will be defined on $\mathbb{C}\left(q^{\frac{1}{2}}\right)$.

Usually, we deal with operators of linear-fractional transformations, as in (3), but sometimes we will have to add the parameter inversion

$$
\tau: q \longmapsto q^{-1},
$$

acting on the functions via $(\tau X)(q)=X\left(q^{-1}\right)$.

\subsection{The operator of imaginary translations}

The main idea of the present paper is to consider the matrix $U \in \operatorname{PSL}(2, \mathbb{Z}[i])$ and its square

$$
U=\left(\begin{array}{cc}
1 & i \\
0 & 1
\end{array}\right), \quad U^{2}=\left(\begin{array}{cc}
1 & 2 i \\
0 & 1
\end{array}\right)
$$

acting on the complex plane $\mathbb{C}$ by imaginary translations, and find a $q$-deformation $U_{q}$, compatible with the $\operatorname{PSL}(2, \mathbb{Z})$-action. We will show the existence of such a deformation and prove its uniqueness in a natural class of linear-fractional operators.

The compatibility with the $q$-deformed $\operatorname{PSL}(2, \mathbb{Z})$-action consists of two properties.

(a) The operator $U_{q}$ must commute with $T_{q}$;

(b) $U_{q}$ must send $[-i]_{q}$ to 0 , and 0 to $[i]_{q}$, where $[-i]_{q}$ and $[i]_{q}$ are as in (9).

Surprisingly, it is much easier to work with the square of the operator $U$. We will prove (in Section 3.1) the following.

Theorem 1.2. There exists a unique element of the group of linear-fractional transformations of $\mathbb{C}\left(q^{\frac{1}{2}}\right)$, commuting with $T_{q}$ and sending $[-i]_{q}$ to $[i]_{q}$ :

$$
\left(U^{2}\right)_{q}=\left(\begin{array}{cc}
1+i\left(q^{\frac{1}{2}}-q^{-\frac{1}{2}}\right) & 2 i q^{-\frac{1}{2}} \\
0 & 1-i\left(q^{\frac{1}{2}}-q^{-\frac{1}{2}}\right)
\end{array}\right) .
$$

It turns out that the "square root" of (12) has a different nature in the following sense. There is no matrix with coefficients depending on $q$ that commutes with $T_{q}$ and sends $[-i]_{q}$ to 0 , and 0 to $[i]_{q}$. In fact, the operator $U_{q}$ inverses the parameter $q$ in the argument.

Definition. The operator $U_{q}$ acts on $\mathbb{C}\left(q^{\frac{1}{2}}\right)$ by the formula

$$
U_{q} X:=\frac{X\left(q^{-1}\right)+i q^{\frac{1}{2}}}{(1-q) X\left(q^{-1}\right)+q}
$$


It is easy to check that $U_{q}$ commutes with $T_{q}$ and that it squares to (12) and satisfies (b). Note also that $U_{q}$ can be written in the matrix form

$$
U_{q}=\left(\begin{array}{cc}
1 & i q^{\frac{1}{2}} \\
1-q & q
\end{array}\right) \circ \tau,
$$

where $\tau$ is the parameter inversion (10).

\section{7 q-deformed Gaussian integers}

Gaussian integers are complex numbers $m+n i$, with $m, n \in \mathbb{Z}$. It is natural to define $q$-deformed Gaussian integers as the orbit of $[0]_{q}=0$ in $\mathbb{C}\left(q^{\frac{1}{2}}\right)$ under the action of the abelian group $\mathbb{Z}^{2}$ generated by the translation operators $T_{q}$ and $U_{q}$.

These $q$-deformed Gaussian integers have quite interesting and nontrivial properties. Their explicit formula (Theorem 4.1) is obtained thanks to a new choice of the parameter:

$$
Q:=\frac{2 i q^{\frac{1}{2}}(q-1)}{q^{2}-q+1}-\frac{q^{2}-3 q+1}{q^{2}-q+1} .
$$

The function $q \mapsto Q(q)$ is quite remarkable. When $q$ is real, $Q$ belongs to the unit circle, i.e., $Q \bar{Q}=1$.

I will also show that $q$-deformed Gaussian integers are related to the Chebyshev polynomials of second kind (Theorem 5.1) in a somewhat unexpected way.

\subsection{The $q$-deformed Picard group and $q$-continued fractions}

The group $\operatorname{PSL}(2, \mathbb{Z}[i])$ of unimodular $2 \times 2$ matrices with Gaussian integer coefficients, usually called the Picard group. This group naturally acts on $\mathbb{Q}[i]$, and we obtain a $q$-deformation of this action defined on $\mathbb{C}\left(q^{\frac{1}{2}}\right)$. It turns out that all of the relations between the generators remain unchanged in the $q$-deformed situation, except for one relation that has no $q$-analog. The "missing" relation makes the complex situation much more complicated than in the real case and leads to an extension of $\operatorname{PSL}(2, \mathbb{Z}[i])$. The $q$-deformation of complex numbers cannot be determined assuming invariance under the full group $\operatorname{PSL}(2, \mathbb{Z}[i])$.

\section{Elliptic points of $\operatorname{PSL}(2, \mathbb{Z})$}

In this short section, we obtain the first information about $q$-deformed complex numbers from the modular invariance. It is due to the fact that some of the (quadratic, i.e., solutions of quadratic equations with integer coefficients) complex numbers, for instance, $i$, are fixed points of real matrices.

\subsection{Modular invariance and fixed points}

I always assume that the $q$-deformation is $\operatorname{PSL}(2, \mathbb{Z})$-invariant.

Since the $q$-deformed generators $T_{q}$ and $S_{q}$ given by (4) and (5) satisfy the same relations (7), the embedding

$$
\operatorname{PSL}(2, \mathbb{Z}) \hookrightarrow \operatorname{PSL}(2, \mathbb{Z}[q])
$$


is well defined and to every $A \in \operatorname{PSL}(2, \mathbb{Z})$ it assigns a matrix whose elements are polynomial in $q$.

For every $x \in \mathbb{Q}$, the $\operatorname{PSL}(2, \mathbb{Z})$-invariance implies

$$
[A(x)]_{q}=A_{q}\left([x]_{q}\right)
$$

If $x$ is a fixed point of $A$, then $[x]_{q}$ has to be a fixed point of $A_{q}$, and can be determined in this way.

\subsection{Elliptic points of $\operatorname{PSL}(2, \mathbb{Z})$}

The standard action of $\operatorname{PSL}(2, \mathbb{Z})$ on $\mathbb{C} \cup\{\infty\}$ has a discrete set of points with nontrivial stabilizer. Such points are called elliptic.

Proposition 2.1. The set of elliptic points of $\operatorname{PSL}(2, \mathbb{Z})$ in the upper half-plane is the $\operatorname{PSL}(2, \mathbb{Z})$-orbit of $i$ and the cube root of 1 :

$$
\left\{i, \frac{-1+\sqrt{3} i}{2}\right\} .
$$

This statement is classical (see, e.g., [16], Chapter VII), let us outline its proof.

Proof. First, one checks that the points (15) are elliptic. Indeed, the imaginary unit $i$ is a fixed point of $S$, while $\frac{-1+\sqrt{3} i}{2}$ is fixed by $S T$.

To prove that any other elliptic point in the upper half-plane is an image of one of the points (15) by an element of $\operatorname{PSL}(2, \mathbb{Z})$, note that $A \in \operatorname{PSL}(2, \mathbb{Z})$ has a fixed point in the upper half-plane if and only if $A$ is an elliptic element of $\operatorname{PSL}(2, \mathbb{Z})$, that is, $\operatorname{tr}(A)=0$ or 1 . In this case, $A$ belongs to the conjugacy class of $S$, or $S T$, respectively.

Remark 2.2. Choose (the standard) fundamental domain of $\operatorname{PSL}(2, \mathbb{Z})$

$$
\mathcal{D}=\left\{z \in \mathbb{C} ; \quad|z|>1,-\frac{1}{2}<\operatorname{Re}(z)<\frac{1}{2}\right\} .
$$

There are no elliptic points points inside any fundamental domain, since $\mathcal{D}$ has the empty intersection with its image under an element of $\operatorname{PSL}(2, \mathbb{Z})$. Therefore, there are exactly three elliptic points on the border of $\mathcal{D}$ :

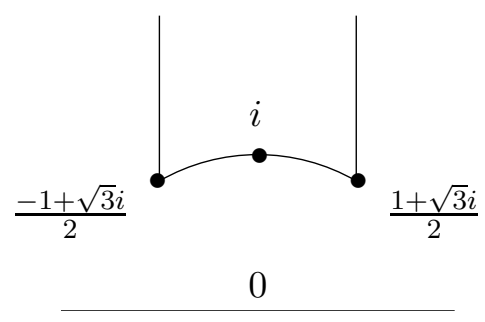

The complex conjugate of the elliptic points are also fixed points (of the same element of $\operatorname{PSL}(2, \mathbb{Z}))$. In the sequel, two points, $i$ and $-i$, will play a crucial role. 


\subsection{The first examples of $q$-deformed complex numbers}

The fixed point of $S_{q}$, of $S_{q} T_{q}$, and of $T_{q} S_{q}$ are easily calculated, they are

$$
[ \pm i]_{q}:= \pm \frac{i}{q^{\frac{1}{2}}}, \quad\left[\frac{-1 \pm \sqrt{3} i}{2}\right]_{q}=\frac{-1 \pm \sqrt{3} i}{2 q}, \quad\left[\frac{1 \pm \sqrt{3} i}{2}\right]_{q}=\frac{1 \pm \sqrt{3} i}{2}
$$

respectively. These are our first examples of $q$-deformed complex numbers.

Remark 2.3. Note that, since the matrix $T_{q} S_{q}$ does not depend of $q$, the points $\frac{1 \pm \sqrt{3} i}{2}$ remain undeformed. Besides 0 and 1 , these are the only numbers with this property. This indicates that the Eisenstein integers (i.e., the numbers of the form $n+m \frac{-1+\sqrt{3} i}{2}$, with $n, m \in \mathbb{Z}$ ) should play some role in our approach. However, in this paper, the considerations are restricted to the Gaussian integers. The only elliptic points of $\operatorname{PSL}(2, \mathbb{Z})$ which are Gaussian integers are $\pm i+n$, where $n \in \mathbb{Z}$.

\section{$3 \quad$ Imaginary translations}

In this section, we define the operator $U_{q}$ acting on the field $\mathbb{C}\left(q^{\frac{1}{2}}\right)$, we will interpret this $U_{q}$ as the $q$-deformation of the operator $U$ of translations by $i$. It turns out that there is no such operator $U_{q}$ in the group of linear-fractional transformation, only its square $U_{q}^{2}$, while $U_{q}$ is more sophisticated.

\subsection{The operator of double imaginary translation}

In this section, we prove Theorem 1.2, Let us start with the general form of the matrix of an operator commuting with the operator $T_{q}$.

Lemma 3.1. The matrix of a linear-fractional operator $A$ on $\mathbb{C}\left(q^{\frac{1}{2}}\right)$, commuting with the operator $T_{q}$ given by (4), is of one of the following two forms

(i) A two-parameter family of triangular matrices

$$
A=\left(\begin{array}{ll}
a & b \\
0 & d
\end{array}\right)
$$

where $a$ and $b$ are arbitrary functions in $q^{\frac{1}{2}}$ and $d=a-(q-1) b$.

(ii) A one-parameter family of matrices proportional to

$$
A=\left(\begin{array}{cc}
1 & (q-1)^{-1} \\
1-q & -1
\end{array}\right)
$$

Proof. For an arbitrary matrix $A=\left(\begin{array}{ll}a & b \\ c & d\end{array}\right)$, one has

$$
T_{q} A=\left(\begin{array}{cc}
q a+c & q b+d \\
c & d
\end{array}\right), \quad A T_{q}=\left(\begin{array}{cc}
q a & a+b \\
q c & c+d
\end{array}\right)
$$


If $c=0$, the condition that $T_{q} A$ and $A T_{q}$ are proportional implies $q b+d=a+b$. If $c \neq 0$, then

$$
q a+c=a, \quad q b+d=q^{-1}(a+b), \quad d=q^{-1}(c+d) .
$$

The first and the third equations give $c=(1-q) a=(q-1) d$, so that $d=-a$. The second equation then leads to $a=(q-1) b$.

The lemma follows.

Suppose now that a triangular matrix $A$ from Lemma 3.1 sends $[-i]_{q}$ to $[i]_{q}$ (where $[-i]_{q}$ and $[i]_{q}$ are as in (9) $)$. One has the following condition

$$
\frac{-a i+b q^{\frac{1}{2}}}{d q^{\frac{1}{2}}}=\frac{i}{q^{\frac{1}{2}}}
$$

so that $(a+d) i=b q^{\frac{1}{2}}$. Substituting $d$ as in Lemma 3.1, one has $2 a=\left(q-1-i q^{\frac{1}{2}}\right) b$. A matrix $A$ of a linear-fractional transformation is defined modulo a scalar multiple, i.e., the coefficient $b$ thus can be chosen in an arbitrary way.

It follows that a matrix $A$ from Lemma 3.1, Part (i), satisfying the conditions of Theorem 1.2, is unique up to a scalar multiple. It is now easy to check that the matrix (12) does satisfy them.

The matrix $A$ from Lemma 3.1, part (ii) does not send $[-i]_{q}$ to $[i]_{q}$.

Theorem 1.2 is proved.

Remark 3.2. Taking $b=2 i q^{-\frac{1}{2}}$, which is natural since we think of $A$ as a $q$-analog of $\left(\begin{array}{cc}1 & 2 i \\ 0 & 1\end{array}\right)$, we obtain the unique matrix satisfying both conditions (commuting with $T_{q}$ and sending $[-i]_{q}$ to $\left.[i]_{q}\right)$, namely

$$
\left(\begin{array}{cc}
1+i\left(q^{\frac{1}{2}}-q^{-\frac{1}{2}}\right) & 2 i q^{-\frac{1}{2}} \\
0 & 1-i\left(q^{\frac{1}{2}}-q^{-\frac{1}{2}}\right)
\end{array}\right)
$$

that we denote by $\left(U^{2}\right)_{q}$.

\subsection{The operator $U_{q}$}

A straightforward attempt to calculate a square root of the operator $\left(U^{2}\right)_{q}$ given by (12) fails. There is no such operator inside the group of linear-fractional transformations.

Lemma 3.3. There is no linear-fractional operator $A$ on $\mathbb{C}\left(q^{\frac{1}{2}}\right)$ commuting with the operator $T_{q}$ and sending $[-i]_{q}$ to 0 , and 0 to $[i]_{q}$.

Proof. Let $A$ be as in Lemma 3.1. The second condition implies two equations

$$
-a i+b q^{\frac{1}{2}}=0, \quad \frac{b}{d}=\frac{i}{q^{\frac{1}{2}}}
$$

Substituting $d=a-(q-1) b$, leads to $(q-1) b=0$, ad so $b=0$, since $q$ is a parameter. But then $A$ has to be zero, which is a contradiction. 
An alternative way to look for a square root of $\left(U^{2}\right)_{q}$ is to adopt the assumption that $U_{q}$ inverses the parameter $q$, as in (10). Let us consider the linear-fractional transformations composed with $\tau$ :

$$
A X:=\frac{a X\left(q^{-1}\right)+b}{c X\left(q^{-1}\right)+d}
$$

where $a, b, c, d$ are some functions in $q$.

Proposition 3.4. The operator (13) is the unique operator on $\mathbb{C}\left(q^{\frac{1}{2}}\right)$ of the form (16) commuting with $T_{q}$ and sending $[-i]_{q}$ to 0 , and 0 to $[i]_{q}$.

Proof. The composition of $A$ with $T_{q}$ is represented by the matrices

$$
T_{q} A=\left(\begin{array}{cc}
q a+c & q b+d \\
c & d
\end{array}\right), \quad A T_{q}=\left(\begin{array}{cc}
q^{-1} a & a+b \\
q^{-1} c & c+d
\end{array}\right)
$$

that are then to be applied to $X\left(q^{-1}\right)$. The operators $A$ and $T_{q}$ commute means that the matrices are proportional. This gives:

$$
c=(1-q) a, \quad d=q a,
$$

with arbitrary $a$ and $b$.

The condition that $A$ sends $[-i]_{q}$ to 0 , and 0 to $[i]_{q}$ reads

$$
b=i q^{\frac{1}{2}} a, \quad b=i q^{-\frac{1}{2}} d .
$$

The obtained system of four equation has a one-parameter family of solutions, namely the coefficients of the matrix

$$
\left(\begin{array}{cc}
a & i q^{\frac{1}{2}} a \\
(1-q) a & q a
\end{array}\right)
$$

Proposition 3.4 is proved.

Let us finally check that the operator $\left(U^{2}\right)_{q}$ given by (12) is, indeed, the square of $U_{q}$.

Proposition 3.5. The composition of $U_{q}$ with itself is the operator (12).

Proof. One checks that

$$
\left(\begin{array}{cc}
1 & i q^{\frac{1}{2}} \\
1-q & q
\end{array}\right) \circ \tau \circ\left(\begin{array}{cc}
1 & i q^{\frac{1}{2}} \\
1-q & q
\end{array}\right) \circ \tau=\left(\begin{array}{cc}
1 & i q^{\frac{1}{2}} \\
1-q & q
\end{array}\right)\left(\begin{array}{cc}
1 & i q^{-\frac{1}{2}} \\
1-\frac{1}{q} & \frac{1}{q}
\end{array}\right)=\left(U^{2}\right)_{q}
$$

This means that the operator (12) is the composition of the operator $U_{q}$ with itself. 


\section{Introducing $q$-deformed Gaussian integers}

Our goal in this section is to calculate explicit formulas and linear recurrences for $q$ deformed Gaussian integers, defined as the orbit of 0 in $\mathbb{C}\left(q^{\frac{1}{2}}\right)$ under the $\mathbb{Z}^{2}$-action generated by $T_{q}$ and $U_{q}$ :

$$
[n i+m]_{q}:=T_{q}^{m} U_{q}^{n}(0)
$$

Since the action of $T$ is given by a simple expression

$$
T^{m}(X(q))=q^{m} X(q)+[m]_{q}
$$

where $[m]_{q}$ is the Euler $q$-integer (11), it suffices to calculate $q$-deformed purely imaginary Gaussian integers $[n i]_{q}$.

To simplify the exposition and immediately explain the nature of these $q$-numbers, let us start with examples, the general formulas and recurrences will be calculated after that.

\subsection{A list of small Gaussian integers}

The first examples are

$$
\begin{aligned}
& {[i]_{q}=i q^{-\frac{1}{2}}} \\
& {[2 i]_{q}=\frac{2 q[i]_{q}}{q^{2}-q+1}-\frac{2(q-1)}{q^{2}-q+1},} \\
& {[3 i]_{q}=-[i]_{q}+\frac{4 q[i]_{q}}{q^{2}-q+1}-\frac{4(q-1)}{q^{2}-q+1},} \\
& {[4 i]_{q}=-\frac{4 q[i]_{q}}{q^{2}-q+1}+\frac{8 q^{2}[i]_{q}}{\left(q^{2}-q+1\right)^{2}}-\frac{8 q(q-1)}{\left(q^{2}-q+1\right)^{2}}} \\
& {[5 i]_{q}=[i]_{q}-\frac{12 q[i]_{q}}{q^{2}-q+1}+\frac{16 q^{2}[i]_{q}}{\left(q^{2}-q+1\right)^{2}}+\frac{4(q-1)}{\left(q^{2}-q+1\right)}-\frac{16 q(q-1)}{\left(q^{2}-q+1\right)^{2}}} \\
& {[6 i]_{q}=\frac{6 q[i]_{q}}{q^{2}-q+1}-\frac{32 q^{2}[i]_{q}}{\left(q^{2}-q+1\right)^{2}}+\frac{32 q^{3}[i]_{q}}{\left(q^{2}-q+1\right)^{3}}} \\
& -\frac{2(q-1)}{\left(q^{2}-q+1\right)}+\frac{16 q(q-1)}{\left(q^{2}-q+1\right)^{2}}-\frac{32 q^{2}(q-1)}{\left(q^{2}-q+1\right)^{3}}, \\
& {[7 i]_{q}=-[i]_{q}+\frac{24 q[i]_{q}}{q^{2}-q+1}-\frac{80 q^{2}[i]_{q}}{\left(q^{2}-q+1\right)^{2}}+\frac{64 q^{3}[i]_{q}}{\left(q^{2}-q+1\right)^{3}}} \\
& -\frac{8(q-1)}{\left(q^{2}-q+1\right)}+\frac{48 q(q-1)}{\left(q^{2}-q+1\right)^{2}}-\frac{64 q^{2}(q-1)}{\left(q^{2}-q+1\right)^{3}}
\end{aligned}
$$




$$
\begin{array}{r}
{[8 i]_{q}=-\frac{8 q[i]_{q}}{q^{2}-q+1}+\frac{80 q^{2}[i]_{q}}{\left(q^{2}-q+1\right)^{2}}-\frac{192 q^{3}[i]_{q}}{\left(q^{2}-q+1\right)^{3}}+\frac{128 q^{4}[i]_{q}}{\left(q^{2}-q+1\right)^{4}}} \\
-\frac{32 q(q-1)}{\left(q^{2}-q+1\right)^{2}}+\frac{128 q^{2}(q-1)}{\left(q^{2}-q+1\right)^{3}}-\frac{128 q^{3}(q-1)}{\left(q^{2}-q+1\right)^{4}} \\
{[9 i]_{q}=[i]_{q}-\frac{40 q[i]_{q}}{q^{2}-q+1}+\frac{240 q^{2}[i]_{q}}{\left(q^{2}-q+1\right)^{2}}-\frac{448 q^{3}[i]_{q}}{\left(q^{2}-q+1\right)^{3}}+\frac{256 q^{4}[i]_{q}}{\left(q^{2}-q+1\right)^{4}}} \\
+\frac{8(q-1)}{\left(q^{2}-q+1\right)}-\frac{112 q(q-1)}{\left(q^{2}-q+1\right)^{2}}+\frac{320 q^{2}(q-1)}{\left(q^{2}-q+1\right)^{3}}-\frac{256 q^{3}(q-1)}{\left(q^{2}-q+1\right)^{4}}
\end{array}
$$

One observes that the coefficients of the imaginary parts $\operatorname{Im}\left([n i]_{q}\right)$ form a triangle that starts as follows

$$
\begin{array}{rccc}
1 & & & \\
2 & & & \\
-1 & 4 & & \\
-4 & 8 & & \\
1 & -12 & 16 & \\
6 & -32 & 32 & \\
-1 & 24 & -80 & 64 \\
\ldots & & &
\end{array}
$$

Quite remarkably, this triangle coincides with the triangle of coefficients of Chebyshev polynomials of second type; see Sequences A008312, A053117 of OEIS [14]. The coefficients of the real part are also connected to the Chebyshev polynomials. The precise connection to Chebyshev polynomials will be explained in Section 5 .

\subsection{The new parameter $Q$}

Let us describe a new choice of the parameter of deformation. Instead of the parameter $q$, we will use the parameter

$$
Q:=\frac{2 i q^{\frac{1}{2}}(q-1)}{q^{2}-q+1}-\frac{q^{2}-3 q+1}{q^{2}-q+1} .
$$

One can understand $Q$ as a formal parameter, but it will also be useful to think of it as a (two-valued) function in $q$.

The parameter $Q$ has several nice properties.

(a) If the initial parameter of deformation $q$ is real, then

$$
Q^{-1}=\bar{Q}
$$

(b) The two-valued function $q \rightarrow Q(q)$ sends the interval $[0,1]$ to two unit half-circles, depending on the choice of the sign of $q^{\frac{1}{2}}= \pm \sqrt{q}$. Below is the positive branch. 


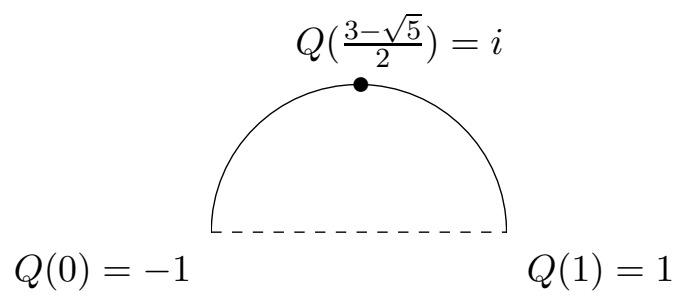

\subsection{The explicit formula and linear recurrence}

Let us give an explicit formula for the $q$-deformed Gaussian integers $[n i]_{q}$.

If $n \in \mathbb{Z}$, we use the standard notation

$$
[n]_{Q}:=\frac{1-Q^{n}}{1-Q}
$$

for Euler's $Q$-integers. As before, the $q$-deformed imaginary unit is $[i]_{q}=i q^{-\frac{1}{2}}$.

The main result of this section is the following.

Theorem 4.1. For every $n \in \mathbb{Z}$, one has

$$
\begin{aligned}
{[2 n i]_{q} } & =[2]_{Q}[n]_{Q}[i]_{q}, \\
{[(2 n-1) i]_{q} } & =\left([2]_{Q}[n]_{Q}-Q^{n}\right)[i]_{q} .
\end{aligned}
$$

Recall also that the general expression for the $q$-deformed Gaussian integers read

$$
[m+n i]_{q}=q^{m}[n i]_{q}+[m]_{q} .
$$

Remark 4.2. More explicitly, the coefficients in (19) and (20) can be rewritten in the form

$$
[2]_{Q}[n]_{Q}=\frac{1-Q^{n}}{1-Q}+\frac{Q-Q^{n+1}}{1-Q}, \quad[2]_{Q}[n]_{Q}-Q^{n}=\frac{1-Q^{n}}{1-Q}+\frac{Q-Q^{n}}{1-Q}
$$

respectively. These are complex rational functions in $q$ that can be calculated substituting (17). Below are some examples of $q$-integers $[n i]_{q}$ rewritten in terms of the parameter $Q$.

\begin{tabular}{c||c|c|c|c|c|c|c}
$n$ & -3 & -2 & -1 & 0 & 1 & 2 & 3 \\
\hline$[n i]_{q}$ & $-\left(1+2 Q^{-1}\right)[i]_{q}$ & $-\left(1+Q^{-1}\right)[i]_{q}$ & $-[i]_{q}$ & 0 & {$[i]_{q}$} & $(1+Q)[i]_{q}$ & $(1+2 Q)[i]_{q}$
\end{tabular}

Proof. One will need the following.

Lemma 4.3. For every $n \in \mathbb{Z}$, the sequence $\left([n i]_{q}\right)_{n \in \mathbb{Z}}$ satisfies the following linear recurrence with constant coefficients

$$
[(n+2) i]_{q}=(Q+1)[n i]_{q}-Q[(n-2) i]_{q} .
$$


Proof of the lemma. This is a direct consequence of the definition of Gaussian $q$-integers. Indeed, one has $[(n+2) i]_{q}=U^{2}\left([n i]_{q}\right)$, where the operator $U^{2}$ is given by (12). One therefore has an affine recurrence

$$
[(n+2) i]_{q}=\frac{1+i\left(q^{\frac{1}{2}}-q^{-\frac{1}{2}}\right)}{1-i\left(q^{\frac{1}{2}}-q^{-\frac{1}{2}}\right)}[n i]_{q}+\frac{2[i]_{q}}{1-i\left(q^{\frac{1}{2}}-q^{-\frac{1}{2}}\right)},
$$

that readily implies (21), with $Q$ as in (17).

Recurrence (21) determines $[n i]_{q}$ from the values $\left([0]_{q},[2 i]_{q}\right)$ and $\left([-i]_{q},[i]_{q}\right)$, for $n$ even and odd, respectively.

Lemma 4.4. The general solution of (21) is of the form

$$
[2 n i]_{q}=\lambda(q) Q^{n}+\mu(q), \quad[(2 n-1) i]_{q}=\tilde{\lambda}(q) Q^{n}+\tilde{\mu}(q)
$$

where $\lambda(q), \mu(q), \tilde{\lambda}(q), \tilde{\mu}(q)$ are arbitrary functions in $q$.

Proof of the lemma. The characteristic polynomial of the linear recurrence (21) is $x^{2}-$ $(Q+1) x+Q$. It has has the solutions: $x=Q$ and $x=1$. Hence (22).

Since $[0]_{q}=0$, and $[2 i]_{q}=(Q+1)[i]_{q}$ (cf. the examples of Section 4.1), we obtain

$$
\lambda(q)=\frac{Q+1}{Q-1}[i]_{q}, \quad \mu(q)=-\frac{Q+1}{Q-1}[i]_{q},
$$

for $n$ even. Substituting these functions to (22) implies (19)).

Similarly, since $[-i]_{q}=-[i]_{q}$, we obtain

$$
\tilde{\lambda}(q)=\frac{2}{Q-1}[i]_{q}, \quad \tilde{\mu}(q)=-\frac{Q+1}{Q-1}[i]_{q},
$$

for $n$ odd, and this entails (20).

Theorem 4.1 is proved.

\subsection{A property of complex conjugation}

Here, again, we assume that $q$ is a real parameter.

Proposition 4.5. The $q$-numbers $[-n i]_{q}$ and $[n i]_{q}$ are complex conjugate of each other:

$$
[-n i]_{q}=\overline{[n i]_{q}} \text {. }
$$

Proof. First, we observe that

$$
[-n i]_{q}(Q)=-[n i]_{q}\left(Q^{-1}\right) .
$$

Indeed, this follows from the form of the recurrence (21) and the fact that this is true for the initial values (see the table above).

Next, (18) implies:

$$
P\left(Q^{-1}\right)=\overline{P(Q)}
$$


for any polynomial $P(Q)$ with real coefficients.

Multiplying any polynomial with real coefficients by purely imaginary $[i]_{q}$, one arrives at the opposite to (23) property:

$$
P\left(Q^{-1}\right)[i]_{q}=-\overline{P(Q)[i]_{q}} .
$$

Hence the result.

\section{5 q-deformed Gaussian integers and Chebyshev polynomials}

In this section, we explain the relationship of imaginary and real parts of $[n i]_{q}$ with the Chebyshev polynomials, experimentally observed in Section 4.1. Our proof is computational, and it would be interesting to have a more conceptual proof.

\subsection{Chebyshev polynomials of second kind}

The classical Chebyshev polynomials of second kind is a sequence of polynomials in one variable satisfying the recurrence

$$
U_{n+1}(x)=2 x U_{n}(x)-U_{n-1}(x)
$$

and the initial conditions

$$
U_{0}(x)=1, \quad U_{1}(x)=2 x .
$$

The sequence of Chebyshev polynomials starts as follows:

$$
\begin{aligned}
& U_{0}(x)=1, \\
& U_{1}(x)=2 x, \\
& U_{2}(x)=4 x^{2}-1, \\
& U_{3}(x)=8 x^{3}-4 x, \\
& U_{4}(x)=16 x^{4}-12 x^{2}+1, \\
& \ldots
\end{aligned}
$$

The well-known determinant formula is

$$
U_{n}(x)=\left|\begin{array}{ccccc}
2 x & 1 & & & \\
1 & 2 x & 1 & & \\
& \ddots & \ddots & \ddots & \\
& & 1 & 2 x & 1 \\
& & & 1 & 2 x
\end{array}\right| .
$$

This tridiagonal determinant is known under the name of continuant, it naturally appears in the theory of continued fractions. 


\subsection{The two "variants" of the Chebyshev polynomials}

Consider a slightly modified recurrence

$$
\tilde{U}_{n+1}(x)=\left\{\begin{array}{rr}
2 x \tilde{U}_{n}(x)-\tilde{U}_{n-1}(x), & n \text { odd }, \\
2 \tilde{U}_{n}(x)-\tilde{U}_{n-1}(x), & n \text { even. }
\end{array}\right.
$$

The initial values will be chosen in one of the following two ways

$$
\tilde{U}_{0}^{I}(x)=1, \quad \tilde{U}_{1}^{I}(x)=2 x, \quad \tilde{U}_{0}^{I I}(x)=1, \quad \tilde{U}_{1}^{I I}(x)=2 .
$$

Hence, for $n$ even we have

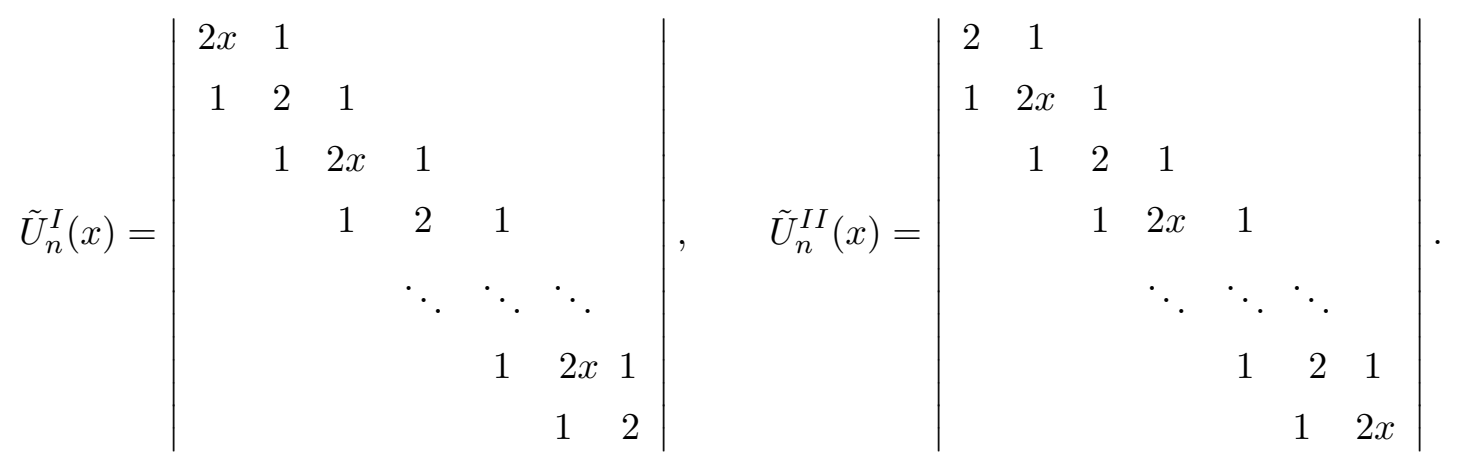

If $n$ is odd, the formulas are similar, with the main diagonal ending with $2 x$ or 2 , respectively.

The sequences of polynomials $\tilde{U}_{n}^{I}(x)$ and $\tilde{U}_{n}^{I I}(x)$ start as follows:

$$
\begin{array}{ll}
\tilde{U}_{0}^{I}(x)=1, & \tilde{U}_{0}^{I I}(x)=1, \\
\tilde{U}_{1}^{I}(x)=2 x, & \tilde{U}_{1}^{I I}(x)=2, \\
\tilde{U}_{2}^{I}(x)=4 x-1, & \tilde{U}_{2}^{I I}(x)=4 x-1, \\
\tilde{U}_{3}^{I}(x)=8 x^{2}-4 x, & \tilde{U}_{3}^{I I}(x)=8 x-4, \\
\tilde{U}_{4}^{I}(x)=16 x^{2}-12 x+1, & \tilde{U}_{4}^{I I}(x)=16 x^{2}-12 x+1, \\
\ldots & \ldots
\end{array}
$$

The polynomials $\tilde{U}_{n}^{I}(x)$ and $\tilde{U}_{n}^{I I}(x)$ have lower degree than the classical Chebyshev polynomials $U_{n}(x)$, but exactly the same coefficients. Note also that $\tilde{U}_{2 m}^{I}(x)=\tilde{U}_{2 m}^{I I}(x)$.

\subsection{Recurrences for the imaginary and real parts of $[n i]_{q}$}

The recurrences and determinant formulas for the sequences of the imaginary and real parts of $[n i]_{q}$ is very similar to that of the Chebyshev polynomials.

Since the imaginary parts of $[n i]_{q}$ are all proportional to $[i]_{q}$, and since the real parts are proportional to $\frac{2(q-1)}{q^{2}-q+1}$, we use the following notation

$$
\mathcal{I}_{n}(z):=\operatorname{Im}\left([n i]_{q}\right)[i]_{q}^{-1}, \quad \mathcal{R}_{n}(z):=-\operatorname{Re}\left([n i]_{q}\right) \frac{q^{2}-q+1}{2(q-1)}
$$


where

$$
z=\frac{q}{q^{2}-q+1}
$$

This considerably simplifies the formulas. It turns out that the coefficients $\mathcal{I}_{n}(z)$ of the imaginary part, and the difference of the coefficients of the real parts, $\mathcal{R}_{n}(z)-\mathcal{R}_{n-2}(z)$, of $[n i]_{q}$ coincide with the variants of the Chebyshev polynomials:

Theorem 5.1. One has

$$
\mathcal{I}_{n+1}(z)=\tilde{U}_{n}^{I}(z), \quad \mathcal{R}_{n+2}(z)-\mathcal{R}_{n}(z)=\tilde{U}_{n}^{I I}(z) .
$$

where $z$ is given by (25).

Proof. This statement follows from the following recurrence.

Lemma 5.2. (i) The imaginary parts of $[n i]_{q}$ satisfy the recurrence

$$
\mathcal{I}_{n+1}(z)= \begin{cases}2 z \mathcal{I}_{n}(z)-\mathcal{I}_{n-1}(z), & n \text { odd } \\ 2 \mathcal{I}_{n}(z)-\mathcal{I}_{n-1}(z), & n \text { even }\end{cases}
$$

(ii) the real parts of $[n i]_{q}$ satisfy the recurrence

$$
\mathcal{R}_{n+1}(z)= \begin{cases}2 z \mathcal{R}_{n}(z)-\mathcal{R}_{n-1}(z)-1, & n \text { odd } \\ 2 \mathcal{R}_{n}(z)-\mathcal{R}_{n-1}(z), & n \text { even }\end{cases}
$$

Proof of the lemma. Our proof is a straightforward computation using the induction on $n$. Let us give the details for the imaginary part of $[n i]_{q}$, in the case where $n$ is even.

One needs to prove that

$$
\mathcal{I}_{n+2}=(4 z-2) \mathcal{I}_{n}-\mathcal{I}_{n-2},
$$

which is equivalent to (27).

Recurrence (21) implies

$$
\begin{aligned}
\operatorname{Im}\left([(n+2) i]_{q}\right)= & (\operatorname{Re}(Q)+1) \operatorname{Im}\left([n i]_{q}\right)-\operatorname{Re}(Q) \operatorname{Im}\left([(n-2) i]_{q}\right) \\
& +\operatorname{Im}(Q)\left(\operatorname{Re}\left([n i]_{q}\right)-\operatorname{Re}\left([(n-2) i]_{q}\right)\right) .
\end{aligned}
$$

For the first line in the right-hand-side,

$$
\begin{array}{r}
(\operatorname{Re}(Q)+1) \operatorname{Im}\left([n i]_{q}\right)-\operatorname{Re}(Q) \operatorname{Im}\left([(n-2) i]_{q}\right)= \\
(1-\operatorname{Re}(Q))\left(\operatorname{Im}\left([n i]_{q}\right)+\operatorname{Im}\left([(n-2) i]_{q}\right)\right)+2 \operatorname{Re}(Q) \operatorname{Im}\left([n i]_{q}\right)-\operatorname{Im}\left([(n-2) i]_{q}\right) .
\end{array}
$$

The first line in the right-hand-side becomes

$$
(1-\operatorname{Re}(Q))\left(\operatorname{Im}\left([n i]_{q}\right)+\operatorname{Im}\left([(n-2) i]_{q}\right)\right)=\frac{4 q(q-1)^{2}}{\left(q^{2}-q+1\right)^{2}} \mathcal{I}_{n-1}[i]_{q}
$$


Indeed, by induction hypothesis, we know that

$$
\mathcal{I}_{n}+\mathcal{I}_{n-2}=\frac{2 q}{q^{2}-q+1} \mathcal{I}_{n-1}
$$

Again, by induction hypothesis,

$$
\operatorname{Re}\left([n i]_{q}\right)-\operatorname{Re}\left([(n-2) i]_{q}\right)=-\frac{2(q-1)}{q^{2}-q+1} \mathcal{I}_{n-1},
$$

for $n$ even, and therefore

$$
\operatorname{Im}(Q)\left(\operatorname{Re}\left([n i]_{q}\right)-\operatorname{Re}\left([(n-2) i]_{q}\right)\right)=-\frac{4 q(q-1)^{2}}{\left(q^{2}-q+1\right)^{2}} \mathcal{I}_{n-1}[i]_{q}
$$

After cancellation, one finally obtains

$$
\mathcal{I}_{n+2}=2 \operatorname{Re}(Q) \mathcal{I}_{n}-\mathcal{I}_{n-2},
$$

but the coefficient $2 \operatorname{Re}(Q)$ equals to $4 z-2$. Hence the first formula in (27).

The other cases are similar.

Part (i) of Lemma 5.2 implies that $\mathcal{I}_{n}$ satisfy recurrence (24). Since the initial values of $\mathcal{I}_{n}$ coincide with those of $\tilde{U}_{n}^{I}(z)$, this implies the first formula (26).

Part (ii) of Lemma 5.2 implies that the difference of the real parts, $\mathcal{R}_{n}-\mathcal{R}_{n-2}$, also satisfies recurrence (24). The initial values of $\mathcal{R}_{n}-\mathcal{R}_{n-2}$ coincide with those of $\tilde{U}_{n}^{I I}(z)$.

Theorem 5.1 follows.

\section{The $q$-deformed Picard group $\operatorname{PSL}(2, \mathbb{Z}[i])$}

The group $\mathrm{SL}(2, \mathbb{Z}[i])$ is called the Picard group. It consists of $2 \times 2$ matrices

$$
A=\left(\begin{array}{ll}
a & b \\
c & d
\end{array}\right), \quad a d-b c=1,
$$

where the coefficients $a, b, c, d$ are Gaussian integers. The group $\operatorname{SL}(2, \mathbb{Z}[i])$ acts on complex rationals by linear-fractional transformations. For $x \in \mathbb{Q}[i] \cup\{\infty\}$, one has

$$
A(x)=\frac{a x+b}{c x+d}
$$

the action is transitive and faithful for the projectivization $\operatorname{PSL}(2, \mathbb{Z}[i]):=\operatorname{SL}(2, \mathbb{Z}[i]) /\{ \pm \operatorname{Id}\}$. The Picard group was an object of many studies since the classical book [2].

Our next goal is to describe the $q$-deformation of the group PSL $(2, \mathbb{Z}[i])$ that naturally arises in our context. I give here only an "esquisse" and believe that this $q$-deformation

of $\operatorname{PSL}(2, \mathbb{Z}[i])$ deserves a further study. 


\subsection{Generators and relations of $\operatorname{PSL}(2, \mathbb{Z}[i])$}

The projective Picard group is generated by the matrices

$$
T=\left(\begin{array}{ll}
1 & 1 \\
0 & 1
\end{array}\right), \quad S=\left(\begin{array}{cc}
0 & -1 \\
1 & 0
\end{array}\right), \quad U=\left(\begin{array}{cc}
1 & i \\
0 & 1
\end{array}\right), \quad L=\left(\begin{array}{cc}
-i & 0 \\
0 & i
\end{array}\right)
$$

with the following relations

$$
\begin{aligned}
T U & =U T, \\
S^{2}=L^{2}=(T L)^{2}=(S L)^{2}=(U L)^{2} & =\mathrm{Id}, \\
(T S)^{3}=(U S L)^{3} & =\mathrm{Id} .
\end{aligned}
$$

Any relation between the generators $R, S, U, L$ is a corollary of the relations (29)-(31) (see [19, 1]). Note, as pointed in [19], that $L$ can be expressed in $R, S, U$, and thus removed from the list of the generators, but the relations between $R, S, U$ become more complicated.

Since an element of $\operatorname{PSL}(2, \mathbb{Z}[i])$ is defined up to a scalar multiple, $L$ can be rewritten as follows

$$
L=\left(\begin{array}{cc}
-1 & 0 \\
0 & 1
\end{array}\right)
$$

Note also that, instead of $L$, one can chose the generator

$$
J=S L=\left(\begin{array}{ll}
0 & 1 \\
1 & 0
\end{array}\right)
$$

which is particularly useful for continued fractions.

\subsection{The operator $L_{q}$}

We already have the $q$-deformed operators $T_{q}, S_{q}, U_{q}$. The remaining generator $L$ and its $q$-deformation also appeared in the context of $q$-deformed rational numbers [11, 13, 8].

The operator of linear-fractional transformations associated with the matrix $L$ is the "negation operator": $L(x)=-x$. It was observed in [11, 13, 8] that, besides the invariance under the modular group action, $q$-deformed rational numbers satisfy one more invariance property:

$$
[-x]_{q}=-q^{-1}[x]_{q^{-1}}
$$

This means that we also have an action of $L$ on the space of rational functions. Let us adopt this action as the definition of $L_{q}$.

Definition. Set

$$
L_{q} X:=-\frac{X\left(q^{-1}\right)}{q}
$$

Similarly to $U_{q}$, the operator (32) inverses the parameter of deformation $q$. Hence $L_{q}$ can be represented by the matrix

$$
L_{q}=\left(\begin{array}{cc}
-1 & 0 \\
0 & q
\end{array}\right) \circ \tau
$$

where $\tau$ is as in (10). 


\subsection{Relations between the $q$-deformed generators}

The four operators $T_{q}, S_{q}, U_{q}, L_{q}$ satisfy all the relations of $\operatorname{PSL}(2, \mathbb{Z}[i])$, except for the last one.

Proposition 6.1. The operators $T_{q}, S_{q}, U_{q}, L_{q}$ satisfy the following relations

$$
\begin{aligned}
T_{q} U_{q} & =U_{q} T_{q}, \\
S_{q}^{2}=L_{q}^{2}=\left(T_{q} L_{q}\right)^{2}=\left(S_{q} L_{q}\right)^{2}=\left(U_{q} L_{q}\right)^{2} & =\mathrm{Id}, \\
\left(T_{q} S_{q}\right)^{3} & =\mathrm{Id} .
\end{aligned}
$$

Proof. All of the relations, except for $\left(U_{q} L_{q}\right)^{2}=\mathrm{Id}$, have already been checked in [11, 8]. Let us give here the details of the computation for the latter relation. The product of the operators $U_{q} L_{q}$ is the linear-fractional transformation given by the matrix

$$
U_{q} L_{q}=\left(\begin{array}{cc}
1 & i q^{\frac{1}{2}} \\
1-q & q
\end{array}\right)\left(\begin{array}{cc}
-1 & 0 \\
0 & q^{-1}
\end{array}\right)=\left(\begin{array}{cc}
-1 & i q^{-\frac{1}{2}} \\
q-1 & 1
\end{array}\right) .
$$

One then obtains

$$
\left(U_{q} L_{q}\right)^{2}=\left(\begin{array}{cc}
1+i q^{\frac{1}{2}}-i q^{-\frac{1}{2}} & 0 \\
0 & 1+i q^{\frac{1}{2}}-i q^{-\frac{1}{2}}
\end{array}\right)
$$

which is the identity matrix up to a scalar multiple.

Note that the fact that all the relations, except for one, are unchanged is quite remarkable. This allows one to control the structure of the obtained group. I do not know if every relation between $T_{q}, S_{q}, U_{q}, L_{q}$ is a corollary of (33)-(35); computer experiments allow us to conjecture that this is, indeed, the case.

\subsection{Extension of the group $\operatorname{PSL}(2, \mathbb{Z}[i])$}

The group generated by the operators $T_{q}, S_{q}, U_{q}, L_{q}$ is a subgroup of the group of matrices with coefficients in $\mathbb{C}\left(q^{\frac{1}{2}}\right)$ composed with $\tau$. This group is an extension of $\operatorname{PSL}(2, \mathbb{Z}[i])$ :

$$
\{1\} \longrightarrow \mathcal{N} \longrightarrow \operatorname{PSL(2,\mathbb {Z}}[i]) \longrightarrow \operatorname{PSL}(2, \mathbb{Z}[i]) \longrightarrow\{1\},
$$

where $\mathcal{N}$ is the normal subgroup characterized by the condition $A \in \mathcal{N}$ if and only if

$$
A=\mathrm{Id}+(q-1) \tilde{A},
$$

where $\tilde{A}$ is an arbitrary element. Let us explain this in some details.

The relation $(U S L)^{3}=$ Id has no $q$-analog. The matrices

$$
U S L=\left(\begin{array}{cc}
-1 & i \\
i & 0
\end{array}\right), \quad(U S L)^{-1}=\left(\begin{array}{cc}
0 & i \\
i & 1
\end{array}\right)
$$


have index 3 in $\operatorname{PSL}(2, \mathbb{Z}[i])$, but for their $q$-deformations

$$
U_{q} S_{q} L_{q}=\left(\begin{array}{cc}
-1 & i q^{-\frac{1}{2}} \\
i q^{\frac{1}{2}} & i q^{-\frac{1}{2}}-i q^{\frac{1}{2}}
\end{array}\right), \quad\left(U_{q} S_{q} L_{q}\right)^{-1}=\left(\begin{array}{cc}
i q^{\frac{1}{2}}-i q^{-\frac{1}{2}} & i q^{-\frac{1}{2}} \\
i q^{\frac{1}{2}} & 1
\end{array}\right)
$$

this is not true. The operators $\left(U_{q} S_{q} L_{q}\right)^{3}$ and $\left(U_{q} S_{q} L_{q}\right)^{-3}$ belong to the normal subgroup $\mathcal{N}$. Indeed, one checks that

$$
\left(U_{q} S_{q} L_{q}\right)^{3}=\mathrm{Id}+(q-1)\left(\begin{array}{cc}
0 & i q^{-\frac{1}{2}} \\
i q^{\frac{1}{2}} & 1-\left(q^{-\frac{1}{2}}-q^{\frac{1}{2}}\right)
\end{array}\right) .
$$

The condition (36) is stable under conjugation, so that the matrices with this property form a normal subgroup.

\subsection{Concluding remarks}

One needs the notion of continued fractions to define a notion of $q$-deformed complex number. This approach was used in the real case [11, and we believe that the Hurwitz continued fractions (see [4, 5, 3]) will lead to an interesting notion of a $q$-deformed complex number.

Let us finally mention that appearance of an extension of the symmetry group is a commun phenomenon in quantization. In the context of Kirillov-Kostant-Souriau geometric quantization (see [7] and references therein) this leads to an extension of the quantized space. Usually, the initial symplectic manifold increases its dimension by one and becomes a contact manifold. Heuristically, we think that the requirement of $\operatorname{PSL}(2, \mathbb{Z}[i])$-invariance of the $q$-deformation of the complex plane $\mathbb{C}$ (which is naturally symplectic) should lead to a three-dimensional space, yet to be understood.

\section{Acknowledgements}

The idea to use the modular group to determine the first $q$-deformed complex numbers is due to Sophie Morier-Genoud; I am grateful to her for many fruitful discussions. It is a pleasure to thank Dimitry Leites, Sergei Tabachnikov and Alexander Veselov for helpful comments and friendly encouragement. I am grateful to the referees for useful and constructive comments. This paper was partially supported by the ANR project ANR-19-CE40-0021.

\section{References}

[1] B. Fine, Algebraic theory of the Bianchi groups. Monographs and Textbooks in Pure and Applied Mathematics, 129. Marcel Dekker, Inc., New York, 1989. viii+249 pp.

[2] R. Fricke, F. Klein, Vorlesungen über die Theorie der automorphen Funktionen. Band 1: Die gruppentheoretischen Grundlagen. Johnson Reprint Corp., New York; B. G. Teubner Verlagsgesellschaft, Stuttg art 1965, xiv+634 pp.

Engl.: Lectures on the theory of automorphic functions. Vol. 1. Classical Topics in Mathematics, 3. Higher Education Press, Beijing, 2017. xxxv+539 pp. 
[3] D. Hensley, Continued fractions. World Scientific Publishing Co. Pte. Ltd., Hackensack, NJ, 2006. xiv+245 pp.

[4] A. Hurwitz, Über die Entwicklung Complexer Grössen in Kettenbrüche, Acta Math. 11 (1887), 187-200.

[5] J. Hurwitz, Über die Reduction der Binären Quadratischen Formen mit Complexen Coefficienten und Variabeln, Acta Math. 25 (1902), 231-290.

[6] V. Kac, P. Cheung, Quantum calculus. Universitext. Springer-Verlag, New York, 2002. $\mathrm{x}+112 \mathrm{pp}$.

[7] A.A. Kirillov, Geometric quantization. Dynamical systems, IV, 139-176, Encyclopaedia Math. Sci.,4, Springer, Berlin, 2001.

[8] L. Leclere, S. Morier-Genoud, q-deformations in the modular group and of the real quadratic irrational numbers, Adv. in Appl. Math. 130 (2021), 102223, 28 pp.

[9] L. Leclere, S. Morier-Genoud, V. Ovsienko, A. Veselov, On radius of convergence of q-deformed real numbers, arXiv:2102.00891,

[10] T. McConville, B.E. Sagan, C. Smyth, On a rank-unimodality conjecture of MorierGenoud and Ovsienko, Discrete Math. 344 (2021), no. 8, 112483, 13 pp.

[11] S. Morier-Genoud, V. Ovsienko, q-deformed rationals and q-continued fractions. Forum Math. Sigma 8 (2020), e13, 55 pp.

[12] S. Morier-Genoud, V. Ovsienko, On q-Deformed Real Numbers, Experimental Mathematics, DOI: 10.1080/10586458.2019.1671922, arXiv:1908.04365.

[13] S. Morier-Genoud, V. Ovsienko, Quantum numbers and q-deformed Conway-Coxeter friezes, Math. Intelligencer 43 (2021), 61-70.

[14] OEIS Foundation Inc., The On-Line Encyclopedia of Integer Sequences, http://oeis.org.

[15] A. Schmidt, Diophantine approximation of complex numbers, Acta Math. 134 (1975), $1-85$.

[16] J.P. Serre, A course in arithmetic. Springer-Verlag, New York-Heidelberg, 1973.

[17] G. Shimura, Introduction to the Arithmetic Theory of Automorphic Functions, Princeton University Press and Iwami Shoten, 1971, Second Edition, 1994.

[18] R. Stanley, Enumerative combinatorics. Volume 1. Second edition. Cambridge Studies in Advanced Mathematics, 49. Cambridge University Press, Cambridge, 2012. xiv +626 pp.

[19] R. G. Swan, Generators and Relations for certain Special Linear Groups, Adv. Math., 6, 1-77 (1971).

[20] S. P. Vokos, B.; Zumino, J. Wess, Analysis of the basic matrix representation of $\mathrm{GL}_{q}(2, \mathbb{C})$, Z. Phys. C 48 (1990), no. 1, 65-74. 